\title{
An approach to performance assessment and fault diagnosis for rotating machinery equipment
}

\author{
Xiaochuang Tao ${ }^{1}$, Chen $\mathrm{Lu}^{1,2^{*}}$, Chuan $\mathrm{Lu}^{1,2}$ and Zili Wang ${ }^{1,2}$
}

\begin{abstract}
Predict and prevent maintenance is routinely carried out. However, how to address the problem of performance assessment maximizing the use of available monitoring data, and how to build a framework that integrates performance assessment, fault detection, and diagnosis are still a significant challenge. For this purpose, this article introduces an approach to performance assessment and fault diagnosis for rotating machinery, including wavelet packet decomposition for extracting energy feature samples from vibration signals acquired during normal and faulty conditions; clustering analysis for demonstrating the separability of the samples; and Fisher discriminant analysis for providing an optimal lower-dimensional representation, in terms of maximizing the separability among different populations, by projecting the samples into a new space. In the new low-dimensional space, the Mahalanobis distance (MD) between the new measurement data and normal population can be calculated for performance assessment. Moreover, this model for performance assessment only requires data to be available in normal conditions and any one of all possible fault conditions, without the necessity for the full life cycle of condition monitoring data. In addition, if monitoring data under different fault conditions are available, the fault mode can be identified accurately by comparing the MDs between the new measurement data and each fault population. Finally, the proposed method was verified to be successful on performance assessment and fault diagnosis via a hydraulic pump test and a ball bearing test.
\end{abstract}

Keywords: Performance assessment, Fault diagnosis, Fisher discriminant analysis, Mahalanobis distance

\section{Introduction}

Currently, driven by the demand to reduce maintenance costs, shorten repair time, and maintain high availability of equipment, maintenance strategies have progressed from breakdown maintenance (fail and fix) to preventive maintenance, then to condition-based maintenance (CBM), and lately toward a prospect of intelligent predictive maintenance (predict and prevent), [1-3]. While the reactively breakdown maintenance and blindly preventive maintenance do sometimes reduce equipment failures, they are more labor intensive, do not eliminate catastrophic failures and cause unnecessary maintenance. This is where CBM steps in. It was reported that $99 \%$ of mechanical failures especially rotating machinery are preceded by noticeable indicators [4]. That is to say, with the exception of abrupt,

\footnotetext{
* Correspondence: luchen@buaa.edu.cn

'School of Reliability and Systems Engineering, Beihang University, Beijing 100191, People's Republic of China

${ }^{2}$ Science \& Technology Laboratory on Reliability \& Environmental Engineering, Beijing 100191, People's Republic of China
}

catastrophic failures, most faults of rotating machinery equipment have progression processes to failure. We can view the deterioration as a two-stage process: the first stage as normal operation, and the second stage as a potential failure $[5,6]$. Of interest here is when the second stage starts and how it develops. CBM attempts to monitor machinery health based on condition measurements that do not interrupt normal machine operation. Rotating machinery is one of the most common classes of machines. Over the past few years, technologies in condition monitoring, fault diagnostics, and prognosis for rotating machinery, which are important aspects in a CBM program, have been receiving more attention. Because fault diagnosis problems can be considered as classification problems [7], Fisher discriminant analysis (FDA), which is studied in detail in the pattern classification literature, has been applied to conduct fault diagnosis. However, at present, its application has mainly been concentrated to industrial process (especially chemical processes), but rarely to rotating machinery 
equipment [8-11]. Moreover, although performance assessment, fault detection, diagnosis, and prognosis have received increased attention with significant progress [12], currently, few methods can realize those purposes alone. In addition, the incomplete data (monitoring data under normal or fault conditions) have rarely been considered as input data to performance assessment and this has yet to be fully utilized. To fill the gaps, this article uses the combination of FDA and Mahalanobis distance (MD) applied to rotating machinery fault diagnosis, which is further extended to performance assessment and fault detection. Thus, a framework integrating performance assessment, fault detection, and diagnosis is built, which not only solves the problem of when the second stage of potential failure starts and how it develops, but because of its data-driven property also shows appropriate potential and provides a functional interface to performance trend prognosis.

Successful clustering can demonstrate the separability of samples and provide preconditions for FDA. To avoid the influence of the dispersibility of sample data acquired during normal and various fault conditions, the analysis of the separability of sample data is indispensable. Cluster analysis can classify samples into corresponding groups based on the measured parameters, and hierarchical cluster analysis (HCA) is the most commonly used clustering tool $[13,14]$. FDA provides an optimal lower dimensional representation, in terms of maximizing the separability among different populations representative of different operational states, by projecting normal and fault populations, and separating them to the limit in the reconstructed space [15-17]. In the reconstructed low-dimensional space, the MD between the new measurement data and the normal population, constructed using normal data, can be calculated for performance assessment. The MD can also be transformed into a normalized confidence value (CV), according to the presupposed threshold. If an abnormal state is detected by performance assessment, the MDs between the new measurement data and the normal and different fault populations are calculated, to identify which population the new data belong to, and thus, the fault mode can be recognized.

The proposed method for performance assessment only needs monitoring data under normal conditions and any one of all possible fault conditions. As for fault diagnosis, if the monitoring data under different fault conditions are available, accurate diagnosis results can be achieved by this model. Moreover, in this model, the algorithm is simple and intuitive and offers good interpretation for the results. The proposed method was also verified to be effective and pragmatic for performance assessment and fault diagnosis via a hydraulic pump test and a ball bearing test.

\section{Methodology for performance assessment and fault diagnosis}

2.1. Wavelet packet decomposition-based feature extraction In practice, the characteristic frequencies of rotating machinery equipment or components are usually distributed in both high- and low-frequency bands. In view of this fact, wavelet packet analysis (WPA) is proposed to construct a more sophisticated method of orthogonal decomposition based on multi-resolution analysis, which can divide the full frequency band into multi-levels, so that each band contains information that is more specific $[18,19]$. Therefore, wavelet packet decomposition is suitable for extracting both low- and high-frequency features. Statistically analyzing all bands of a signal decomposed by wavelet packet, an energy index of each frequency band can be extracted.

The determination of the wavelet packet decomposition scale is an issue that cannot be ignored. If the decomposition scale is too little, the fault features cannot effectively be extracted, whereas too many scales will increase the dimension of the feature vector, and consequently, the calculating rate can be affected [20,21]. Therefore, in the hydraulic pump and ball bearing performance assessments, according to the frequency spectrum analysis of the vibration signal explained in Section 3, eight frequency band energy indexes $E_{3 j}$ can be calculated by three-layer decomposition.

$$
E_{3 j}=\int\left|S_{3 j}(t)\right|^{2} d t=\sum_{k=1}^{n}\left|x_{j k}\right|^{2}
$$

where $x_{j k}(j=0,1, \ldots, 7 ; k=1,2, \ldots, n)$ denotes the amplitude of discrete points in the reconstructed signal $S_{3 j}$.

When a hydraulic pump or bearing progresses into a state of degradation, the energy of each frequency band will have a great impact, and therefore, the energy can be normalized into a feature vector $T$.

$$
\begin{aligned}
& T=\left[E_{30} / E, E_{31} / E, E_{32} / E, E_{33} / E, E_{34} / E, E_{35} / E, E_{36} / E, E_{37} / E\right] \\
& E=\left(\sum_{j=0}^{7}\left|E_{3 j}\right|^{2}\right)^{1 / 2}
\end{aligned}
$$

\subsection{Separability analysis of sample data}

In order to avoid the influence of the dispersibility of sample data acquired during normal and various fault conditions, analysis of the separability of the sample data is indispensable.

Clustering is an unsupervised pattern classification method, in which the goal is to determine a finite set of categories to describe a dataset according to similarities 
among its objects [22,23]. As one of the most popular clustering methods, HCA consists of mathematically treating each sample as a point in multidimensional space described by the chosen variables; it builds a nested partition set called a cluster hierarchy. When a given sample is taken as a point in the space defined by the variables, the distance between this point and all the other points can be calculated, thereby establishing a matrix that describes the proximity between all the samples studied. Based on this matrix of proximity between the samples, one can construct a similarity diagram called a dendrogram. There are many ways of mathematically grouping these points in multidimensional space in order to form hierarchical clusters [24-26].

\subsection{FDA}

As an optimal linear dimensionality reduction technique, in terms of maximizing the separation between different populations, FDA has been studied in detail in the pattern classification literature [27-29]. For either performance assessment or fault diagnosis, data collected from the unit during normal and various fault states are categorized into different populations, where each population contains data representing a particular state.

\subsubsection{Definition (MD)}

Given the covariance matrix of a $p$-variate population $G$ as $\sum\left(\sum>0\right)$, and $x, y$ are two samples taken from $G$. Defining

$$
d^{2}(x, y)=(x-y)^{\prime} \sum^{-1}(x-y)
$$

Then $d(x, y)$ is called the MD between $x$ and $y$. Defining

$$
d^{2}(x, G)=(x-\mu)^{\prime} \sum^{-1}(x-\mu)
$$

where $\mu$ is the mean vector of $G$, then $d(x, G)$ is called the MD between $x$ and the population $G$.

\subsubsection{Lemma}

Given $A$ is a $p$-order symmetric matrix, and $B>0$ is a $p$-order positive definite matrix, the eigenvalues of $B^{-1} A$ are $\lambda_{1} \geq \lambda_{2} \geq \cdots \geq \lambda_{p}$ and the corresponding standard eigenvectors are $a_{1}, a_{2}, \ldots, a_{p}$ (standardized into $a_{i} \prime B a_{i}=1$ ). Then, the optimization problem is described as below.

$$
\left(P_{1}\right) \quad\left\{\begin{array}{l}
x^{\prime} A x \rightarrow \max \\
x^{\prime} B x=1
\end{array}\right.
$$

when $x=a_{1}$, the maximum value can be achieved as $\lambda_{1}$.

$$
\left(P_{k}\right)\left\{\begin{array}{l}
x^{\prime} A x \rightarrow \max \\
x^{\prime} B x=1, x^{\prime} B a_{i}=0, \\
i=1,2, \ldots, k-1
\end{array}\right.
$$

when $x=a_{k}$, the maximum value can be achieved as $\lambda_{k}$.
For a more detailed discussion of the extremal problem of the quadratic form, refer to [30].

The mathematical derivation process of FDA follows.

Define $G_{1} \sim\left(\mu_{1}, \sum_{1}\right), G_{2} \sim\left(\mu_{2}, \sum_{2}\right), \ldots, G_{k} \sim\left(\mu_{k}, \sum_{k}\right)$ as $k$ populations, where $\mu_{\mathrm{i}}$ and $\sum_{i}$ are, respectively, the mean vector and covariance matrix of $G_{i} \cdot x \in R^{p}$ is a sample to be determined. Through a linear combination of variable indexes in each population, corresponding onedimensional sample $y=a / x$ can be achieved, which may come from any one of those populations $G_{1}^{*} \sim\left(a^{\prime} \mu_{1}, a^{\prime}\right.$ $\left.\sum_{1} a\right), G_{2}^{*} \sim\left(a^{\prime} \mu_{2}, a^{\prime} \sum_{2} a\right), \ldots, G_{k}^{*} \sim\left(a^{\prime} \mu_{k}, a^{\prime} \sum_{k} a\right)$. Then, defining $B_{0}$ and $E_{0}$ as below

$$
\begin{aligned}
B_{0} & =\sum_{i=1}^{k} n_{i}\left(a^{\prime} \mu_{i}-a^{\prime} \bar{\mu}\right)^{2} \\
& =a^{\prime}\left[\sum_{i=1}^{k} n_{i}\left(\mu_{i}-\bar{\mu}\right)\left(\mu_{i}-\bar{\mu}\right)^{\prime}\right] a=a^{\prime} B a \\
E_{0} & =\sum_{i=1}^{k} a^{\prime} \sum_{i} a=a^{\prime}\left(\Sigma_{1}+\Sigma_{2}+\ldots+\Sigma_{k}\right) a=a^{\prime} E a
\end{aligned}
$$

where $\bar{\mu}=\frac{1}{n} \sum_{i=1}^{k} n_{i} \mu_{i}, \quad B=\sum_{i=1}^{k} n_{i}\left(\mu_{i}-\bar{\mu}\right)\left(\mu_{i}-\bar{\mu}\right)^{\prime}$, $E=\sum_{i=1}^{k} \Sigma_{i}, n_{i}$ is the number of samples for $G_{i}, n$ is the total number of samples. Then $B$ is the between-classscatter matrix, and $E$ is the within-class-scatter matrix.

Thinking along the lines of variance analysis, in order to better separate each population, the choice of $a$ should make $B_{0}$ expand as far as possible, while $E_{0}$ narrows as far as possible.

Thus, the first FDA vector $a$ can be determined as

$$
\max _{a}\left(\frac{B_{0}}{E_{0}}=\frac{a^{\prime} B a}{a^{\prime} E a}\right)
$$
lem.

This is equivalent to the following optimization prob-

$$
\text { (P) }\left\{\begin{array}{l}
a^{\prime} B a \rightarrow \max \\
a^{\prime} E a=1
\end{array}\right.
$$

According to the lemma given in Section 2.3.2, when $a$ is the standard eigenvector $a_{1}$ (standardized into $\left.a_{i}^{\prime} B a_{i}=1\right)$ corresponding to the maximum eigenvalue $\lambda_{1}$ of $E^{-1} B$, formula (10) achieves the maximum $\lambda_{1}$. Thus, the first canonical variable is $y_{1}=a_{1}^{\prime} x$.

The second FDA vector is computed to maximize the scatter between classes, while minimizing the scatter within classes, among all axes perpendicular to the first FDA vector $a_{1}$. According to the lemma given in Section 2.3.2, the second canonical variable is $y_{2}=a_{2} \prime x$ and so on for the remaining FDA vectors and canonical variables. Usually, given the first $m$ eigenvalues as $\lambda_{1} \geq \lambda_{2}$ $\geq \cdots \geq \lambda_{m}$, the corresponding standard eigenvectors as $a_{1}$, 


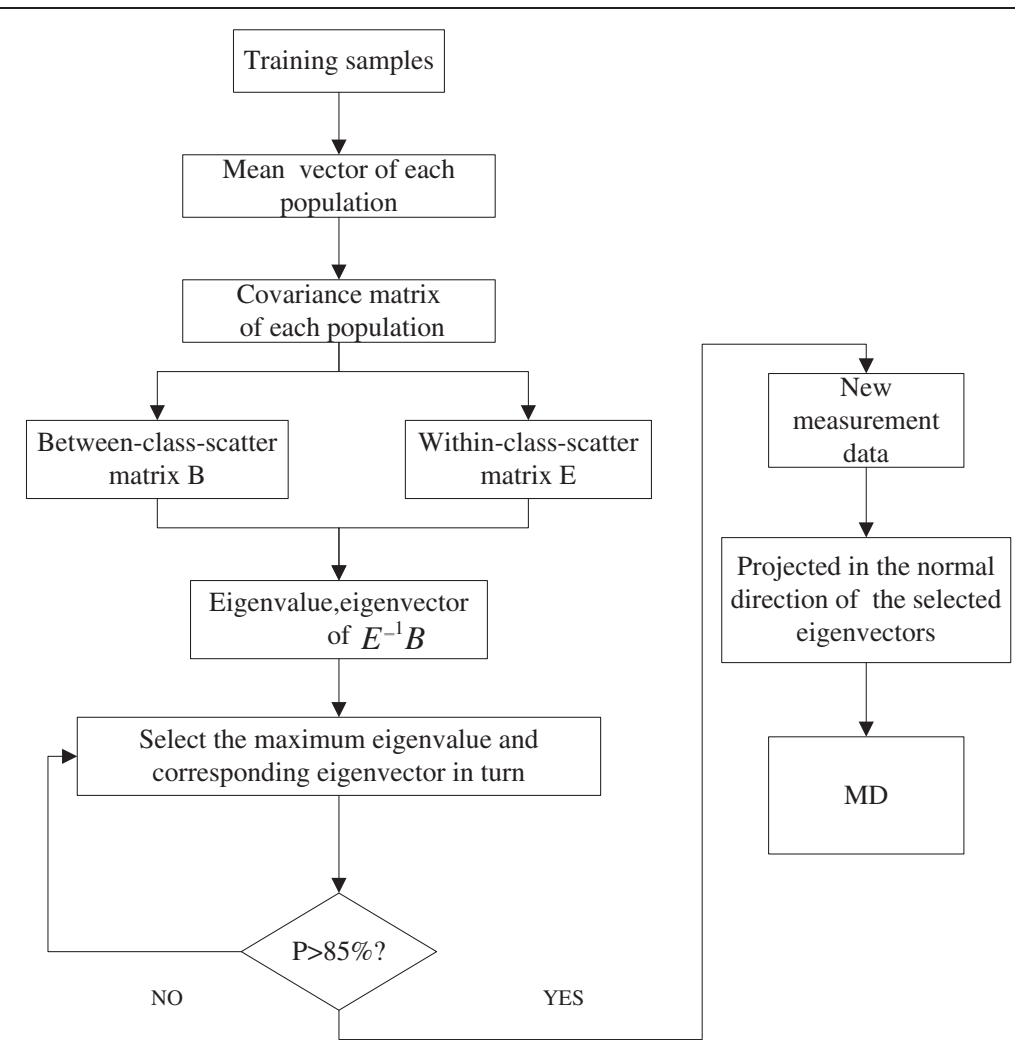

Figure 1 Logic diagram of FDA.
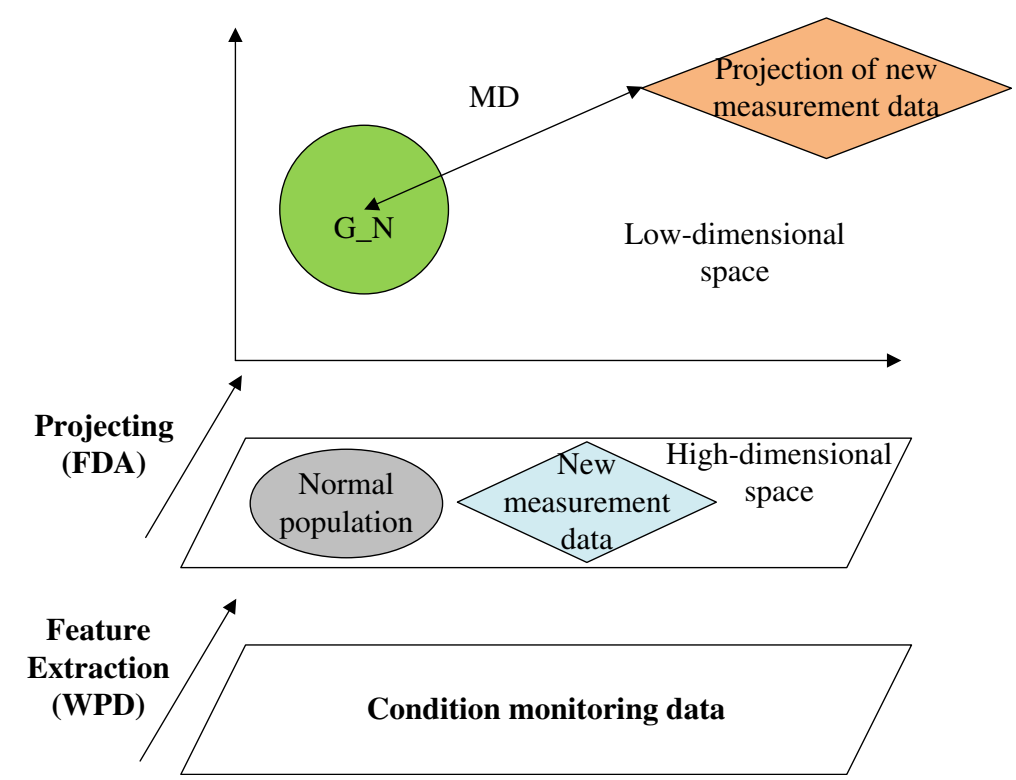

Figure 2 FDA map for performance assessment. 


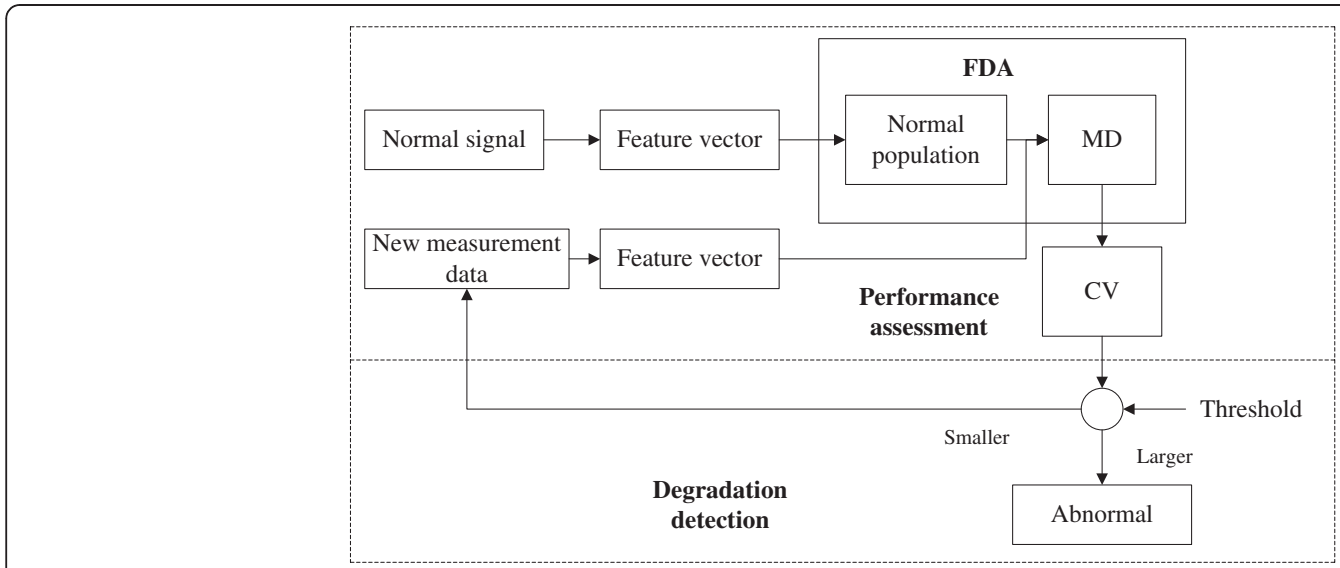

Figure 3 Performance assessment process based on FDA \& MD.

$a_{2}, \ldots, a_{m}$, when the accumulation contribution $P=$ $\frac{\lambda_{1}+\lambda_{2}+\ldots+\lambda_{m}}{\lambda_{1}+\lambda_{2}+\ldots+\lambda_{p}}$ reaches a threshold (such as $85 \%$ ), we can get $m$ unrelated canonical variables $y_{1}, y_{2}, \ldots, y_{m}, y_{m}=a_{m} \prime x$ for discriminant analysis. This is equivalent to mapping a variable from $p$-dimensional space to $m$-dimensional space for analysis, where $m<p$.

After dimensionality reduction, according to the definition given in Section 2.3.1, the MD $d\left(x, G_{j}\right)$ between $x$ and $G_{j}$ can be achieved by calculating the distance between $y=\left(y_{1}, y_{2}, \ldots, y_{m}\right)^{\prime}$ and $G_{j}^{*}(j=1,2, \ldots, k)$. The logic process of FDA is shown as Figure 1.

\subsection{MD for performance assessment}

In practice, the whole life cycle of condition-monitoring data acquired from a machine is already scarce due to irregular measurement recording, and/or the huge amount of time they take to accumulate. For example, a bearing may last several years even under harsh operating conditions. Therefore, regardless of whether it is an experimental or practical application, it is hard to acquire conditionmonitoring data that are representative of the whole life cycle. More commonly, what we can get are incomplete data, such as normal data and fault data [3,31]. Usually, the incomplete data are rarely to be considered as input data of performance assessment, and this has yet to be fully utilized. Consequently, maximizing the use of available data to address the problem of performance assessment is a significant challenge.

As previously mentioned, even if only datasets under normal conditions and any one of all possible fault conditions are available, the normal population can still

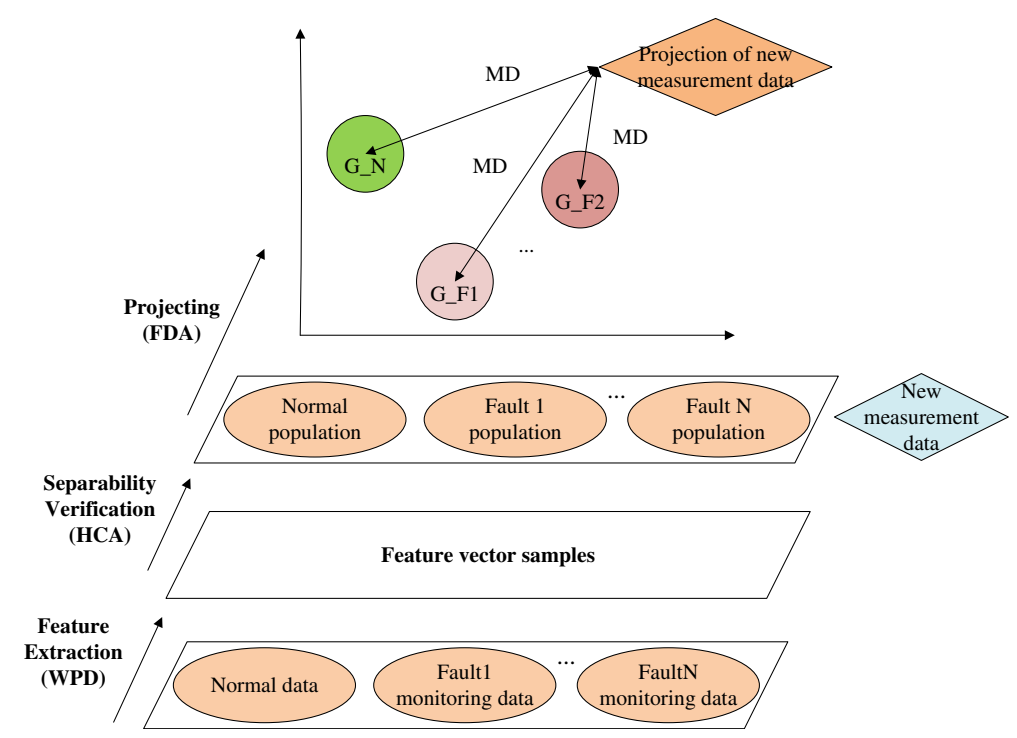

Figure 4 FDA map for fault diagnosis. 


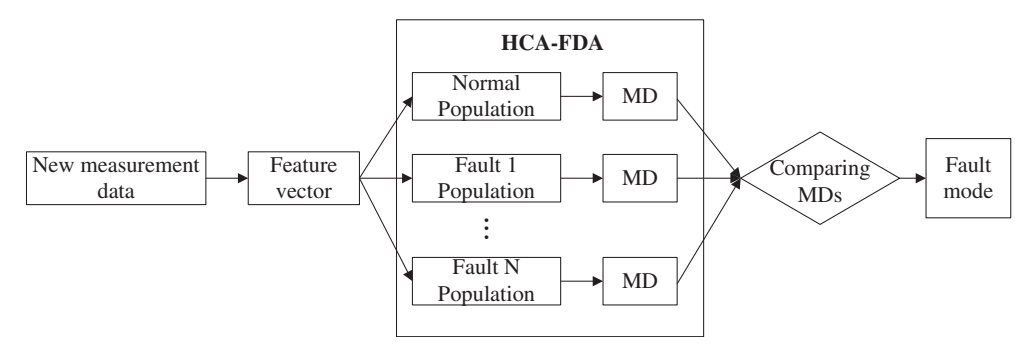

Figure 5 Fault diagnosis process based on FDA \& MD.

accurately be clustered and characterized. As shown in Figure 2, using FDA, a space conversion method can be achieved in which, the normal population and new measurement data can be projected from the original highdimensional space into a new low-dimensional space. Thus, the issue on performance assessment can be addressed by the MD away from the normal population [32]. The MD is calculated between the feature vector extracted from online monitoring data and the normal population and thus, the MD, which indicates how far the input data deviate from the region of normal conditions, can reveal the current performance state. If it exceeds the predetermined threshold (the threshold value can be determined based on engineering experience), the process is probably in an abnormal state. The MD can be defined as

$$
\mathrm{MD}=d\left(x, G_{\text {normal }}\right)
$$

where $x$ is an input feature vector, and $G_{\text {normal }}$ is the normal population.

The performance can be quantized and visualized by following the novel pattern of MD. However, the MD is only an absolute index, without a reference index, it would be difficult to determine whether the current performance condition was good or bad. To succinctly describe the current performance state, the MD coupling with a benchmark can be transformed into a normalized $\mathrm{CV}$, ranging from 0 to 1 . A higher $\mathrm{CV}$ closer to 1 indicates a performance state closer to normal, while a lower $\mathrm{CV}$ closer to 0 is closer to a condition of failure.

$$
\mathrm{CV}=e^{-\frac{\sqrt{\mathrm{MD}}}{c}}
$$

where $c$ is a scale parameter, which is determined by the averaged MDs under normal state and a predetermined CV benchmark.

In summary, the process of performance assessment is shown in Figure 3.

\subsection{MD for fault diagnosis}

If monitoring data under different fault conditions are available, several groups of feature vector samples can be extracted as a learning set. As shown in Figures 4 and 5,

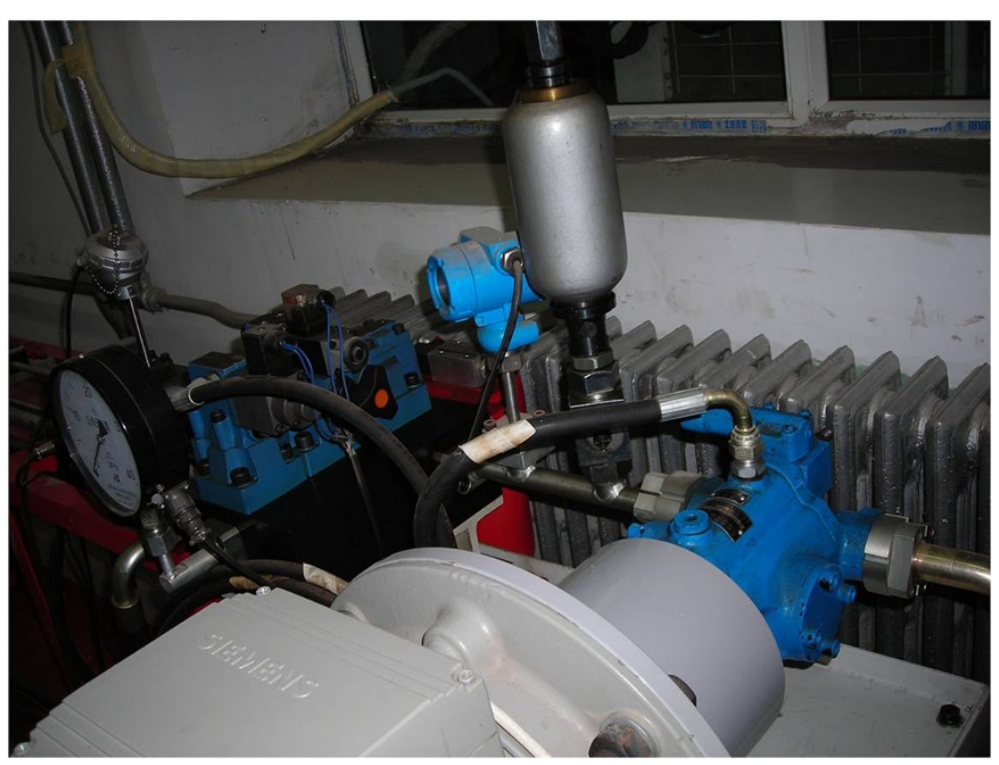

Figure 6 Test-rig of hydraulic pump. 


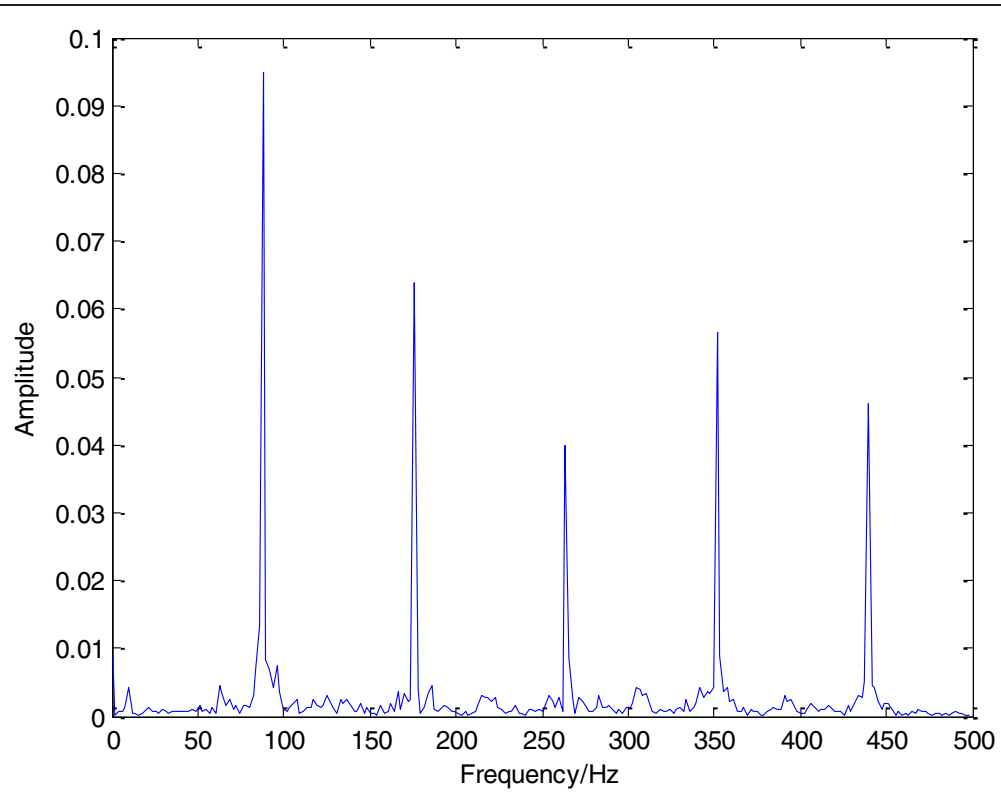

Figure 7 FFT spectrum of acquired normal signal.

with $\mathrm{HCA}$, the learning set will be classified into different populations corresponding to the original operational states. On the basis of demonstration of the separability of feature vector samples, those clustered populations representative of different states, including normal and faults, are analyzed by FDA. FDA provides an optimal lowerdimensional representation in terms of maximizing the separability among different populations. In the new low- dimensional space, the MDs between the real measurement data and those different populations can be calculated, according to the discriminant rule as follows

If $d^{2}\left(x, G_{l}\right)=\min _{1 \leq j \leq k} d^{2}\left(x, G_{j}\right)$, then $x \in G_{l}$.

The state represented by the population that has the minimum MD with the real measurement data can be identified as the current operating state and thus fault diagnosis is completed.

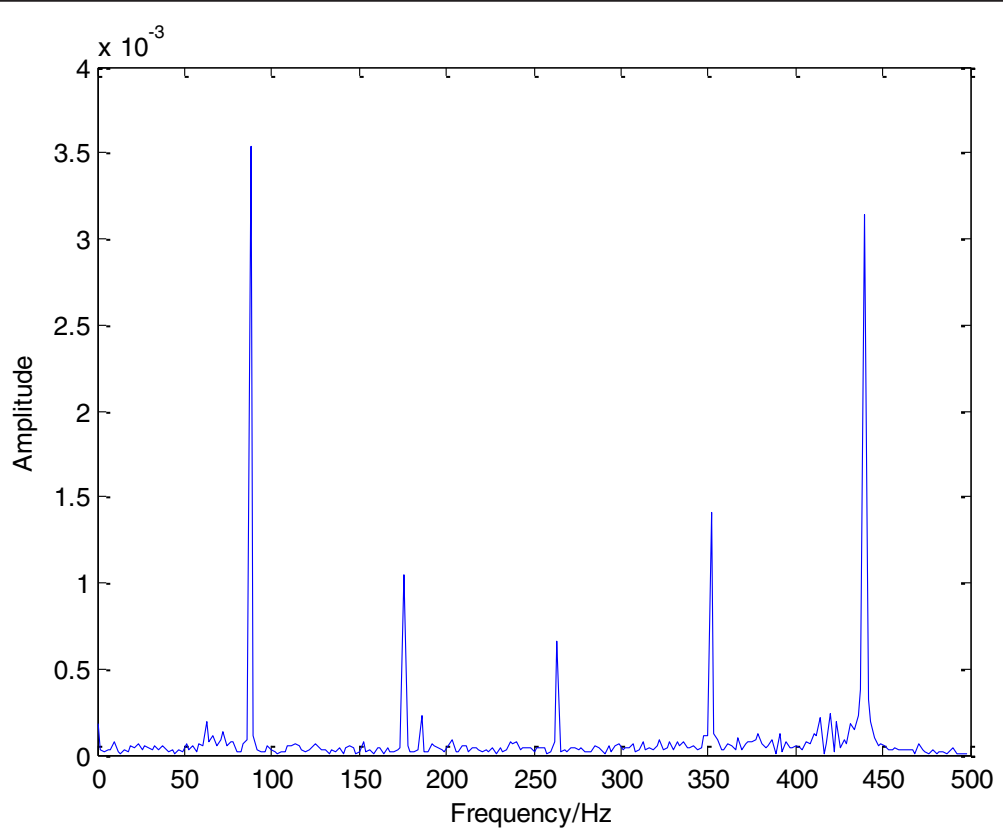

Figure 8 FFT spectrum of acquired fault (slipper loose) signal. 
Table 1 Feature vector samples for learning (hydraulic pump)

\begin{tabular}{lllllllll}
\hline Number & $\mathbf{1}$ & $\mathbf{2}$ & $\mathbf{3}$ & $\mathbf{4}$ & $\mathbf{5}$ & $\mathbf{6}$ & $\mathbf{7}$ \\
\hline Normal_1 & 0.0304 & 0.8484 & 0.1399 & 0.3570 & 0.1182 & 0.1565 & 0.1143 \\
\hline Normal_2 & 0.0343 & 0.8433 & 0.1396 & 0.3557 & 0.1061 & 0.1819 & 0.1085 & 0.2843 \\
\hline Normal_3 & 0.0305 & 0.8307 & 0.1360 & 0.3732 & 0.1231 & 0.1659 & 0.1228 & 0.2926 \\
\hline Normal_4 & 0.0280 & 0.8163 & 0.1654 & 0.3778 & 0.1183 & 0.1491 & 0.1432 \\
\hline Fault1_1 & 0.0256 & 0.8182 & 0.0260 & 0.1312 & 0.3582 & 0.4088 & 0.0290 \\
\hline Fault1_2 & 0.0329 & 0.8141 & 0.0310 & 0.1312 & 0.3450 & 0.4265 & 0.0355 & 0.3256 \\
\hline Fault1_3 & 0.0355 & 0.8256 & 0.0378 & 0.1357 & 0.3483 & 0.3972 & 0.0323 \\
\hline Fault1_4 & 0.0329 & 0.8266 & 0.0358 & 0.1284 & 0.3950 & 0.3517 & 0.0341 \\
\hline Fault2_1 & 0.0261 & 0.2428 & 0.5679 & 0.0871 & 0.0190 & 0.0361 & 0.1309 \\
\hline Fault2_2 & 0.0156 & 0.2385 & 0.5717 & 0.0861 & 0.0229 & 0.0366 & 0.1785 \\
\hline Fault2_3 & 0.0193 & 0.2405 & 0.5628 & 0.0862 & 0.0278 & 0.0283 & 0.7775 \\
\hline Fault2_4 & 0.0116 & 0.2479 & 0.5393 & 0.0816 & 0.0275 & 0.0319 & 0.0507 \\
\hline
\end{tabular}

\section{Experimental verification}

Two experimental cases (i) hydraulic pump performance assessment and fault diagnosisand (ii) ball bearing performance assessment and fault diagnosis are presented to validate the effectiveness and practicality of applying the proposed method to performance assessment and fault diagnosis of rotating machinery equipment. The description of these case studies will follow the sequence: (1) experimental setup and data acquisition; (2) signal analysis and feature extraction; (3) analysis of performance assessment and fault diagnosis results.

\subsection{Performance assessment and fault diagnosis for hydraulic pump}

The hydraulic pump is the heart of a hydraulic system, which determines whether the whole system can run normally or not. Therefore, performance assessment and fault diagnosis of the hydraulic pump is of great importance. Usually, when a hydraulic pump is under an abnormal state, it will be revealed by changes of vibration.
Because most mechanical faults are reflected by vibration [33], the vibration signals of the hydraulic pump are collected and analyzed in this experiment for performance assessment and fault diagnosis.

\subsubsection{Experimental setup and data acquisition}

In this experiment, a hydraulic pump was tested and analyzed, as shown in Figure 6. Two commonly occurring faults in hydraulic pumps were set: slipper loose and valve plate wear. Under different states (normal, faults), monitoring data (vibration signal) were, respectively, acquired from the hydraulic pump end using an acceleration transducer, with motor speed stabilized at $528 \mathrm{rpm}$, and a sampling frequency of $1000 \mathrm{~Hz}$.

\subsubsection{Feature extraction by WPA}

To determine the wavelet packet decomposition scale, FFT was implemented for the vibration signals acquired under normal, slipper loose, and valve plate wear states. Through the analysis of the frequency spectrum, as shown

Table 2 Feature vector samples for testing (hydraulic pump)

\begin{tabular}{lllllllll}
\hline Number & $\mathbf{1}$ & $\mathbf{2}$ & $\mathbf{3}$ & $\mathbf{4}$ & $\mathbf{5}$ & $\mathbf{6}$ & $\mathbf{7}$ & $\mathbf{8}$ \\
\hline N_1 & 0.0428 & 0.8110 & 0.1647 & 0.4138 & 0.1297 & 0.1359 & 0.1495 & 0.2908 \\
\hline N_2 & 0.0300 & 0.8195 & 0.1626 & 0.4070 & 0.1180 & 0.1437 & 0.1434 \\
\hline N_3 & 0.0353 & 0.8078 & 0.1836 & 0.4182 & 0.1089 & 0.1534 & 0.1464 & 0.2833 \\
\hline N_4 & 0.0326 & 0.8142 & 0.1716 & 0.4177 & 0.1107 & 0.1467 & 0.1538 & 0.2732 \\
\hline F1_1 & 0.0293 & 0.8259 & 0.0367 & 0.1255 & 0.4254 & 0.3138 & 0.0316 \\
\hline F1_2 & 0.0243 & 0.8344 & 0.0266 & 0.1398 & 0.3832 & 0.3446 & 0.0229 & 0.1397 \\
\hline F1_3 & 0.0318 & 0.8399 & 0.0281 & 0.1274 & 0.3242 & 0.3902 & 0.0266 \\
\hline F1_4 & 0.0360 & 0.8293 & 0.0338 & 0.1308 & 0.4289 & 0.2984 & 0.0237 \\
\hline F2_1 & 0.0088 & 0.2475 & 0.5249 & 0.0839 & 0.0189 & 0.0374 & 0.1356 \\
\hline F2_2 & 0.0185 & 0.2501 & 0.5710 & 0.0907 & 0.0299 & 0.0232 & 0.1383 \\
\hline F2_3 & 0.0095 & 0.2463 & 0.5446 & 0.0841 & 0.0256 & 0.0263 & 0.7945 \\
\hline F2_4 & 0.0102 & 0.2476 & 0.5235 & 0.0883 & 0.0236 & 0.0308 & 0.0484 \\
\hline
\end{tabular}




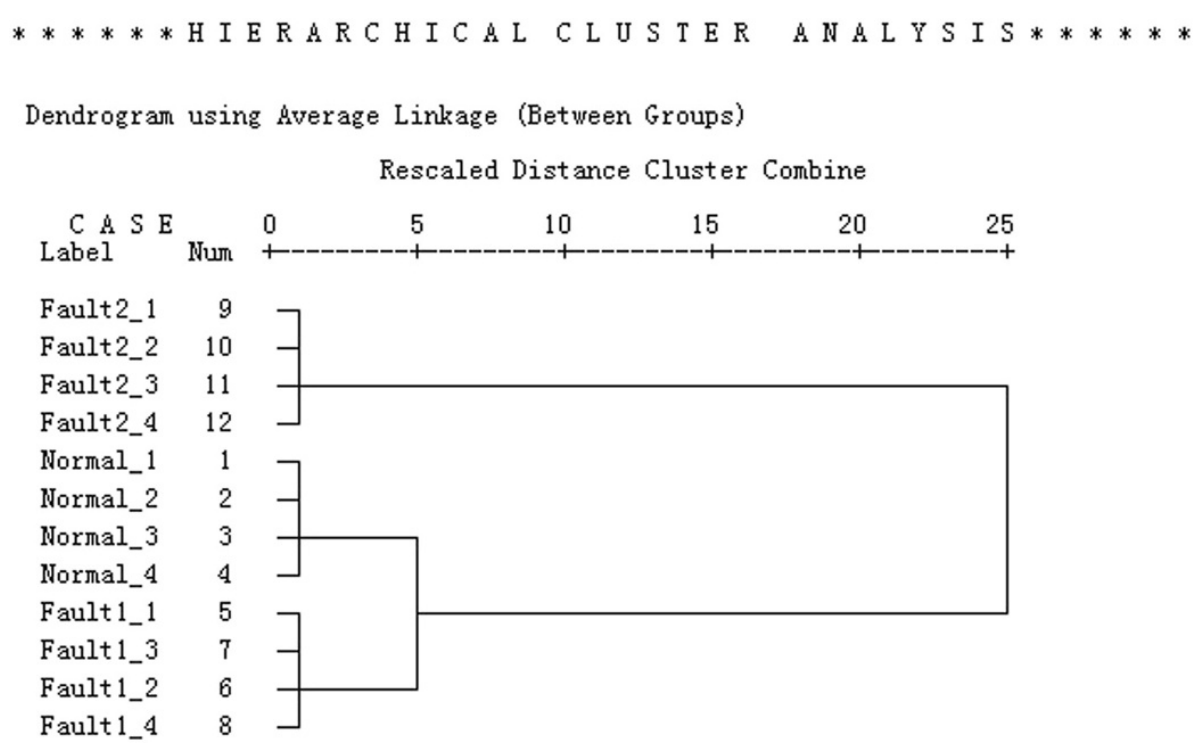

Figure 9 A tree dendrogram of HCA (hydraulic pump).

in Figures 7 and 8, it can be seen that there are clear peaks appearing evenly at six characteristic frequencies. Furthermore, the normal signal and fault (slipper loose) signal have distinctively different peaks at the six characteristic frequencies. Therefore, each set of acquired vibration signals is suitable to be decomposed into eight frequency bands by three-layer wavelet packet decomposition. In that way, all the peaks can be contained in different frequency bands. Then, an eight-dimensional feature vector can be constructed by calculating and normalizing the energy of each band. The frequency range corresponding to each frequency band is $\left(n \omega_{\max } / 2^{N},(n+1) \omega_{\max } / 2^{N}\right)$, where $N=3, n=0,1, \ldots, 7$, and $\omega_{\max }$ is the maximum frequency; here $\omega_{\max }=498$.

For those three states, eight feature vector samples were acquired, respectively. The first four samples of each state were used as the FDA learning set (for clearer identification they were numbered as Normal_1 to Normal_4, Fault1_1 to Fault1_4, and Fault2_1 to Fault2_4, corresponding to the normal state, slipper loose state, and valve plate wear conditions, respectively), while the others were used as the testing set (they were also numbered in the same manner as: N_1 to N_4, F1_1 to F1_4, and F2_1 to F2_4, respectively).

The feature vector samples for learning and testing are shown in Tables 1 and 2.

\subsubsection{Analysis of states separability}

In order to demonstrate the separability of feature vector samples acquired during normal, slipper loose, and valve plate wear states, HCA was selected and carried out on the learning set. As shown in Figure 9, the tree dendrogram clearly shows the whole process of clustering. First, each sample was taken as a class. After the first clustering, according to the distance between classes, samples Fault2_1 to Fault2_4 were merged into a cluster, samples Normal_1 to Normal_4 were merged into another cluster, and samples Fault1_1 to Fault1_4 were merged into the third cluster. Moreover, from the cluster membership, as shown in Table 3, it was found that all the normal samples gathered in cluster 1 , all the slipper loose samples gathered in cluster 2 , and all the valve plate wear samples gathered in cluster 3. The results are consistent perfectly with the practical situation, and can be seen as strong evidence for the separability of the different states.

Table 3 Clustering membership of learning set (hydraulic pump)

\begin{tabular}{lc}
\hline Case & Cluster index \\
\hline 1:Normal_1 & 1 \\
\hline 2:Normal_2 & 1 \\
\hline 3:Normal_3 & 1 \\
\hline 4:Normal_4 & 1 \\
\hline 5:Fault1_1 & 2 \\
\hline 6:Fault1_2 & 2 \\
\hline 7:Fault1_3 & 2 \\
\hline 8:Fault1_4 & 2 \\
\hline 9:Fault2_1 & 3 \\
\hline 10:Fault2_2 & 3 \\
\hline 11:Fault2_3 & 3 \\
\hline 12:Fault2_4 & 3 \\
\hline
\end{tabular}




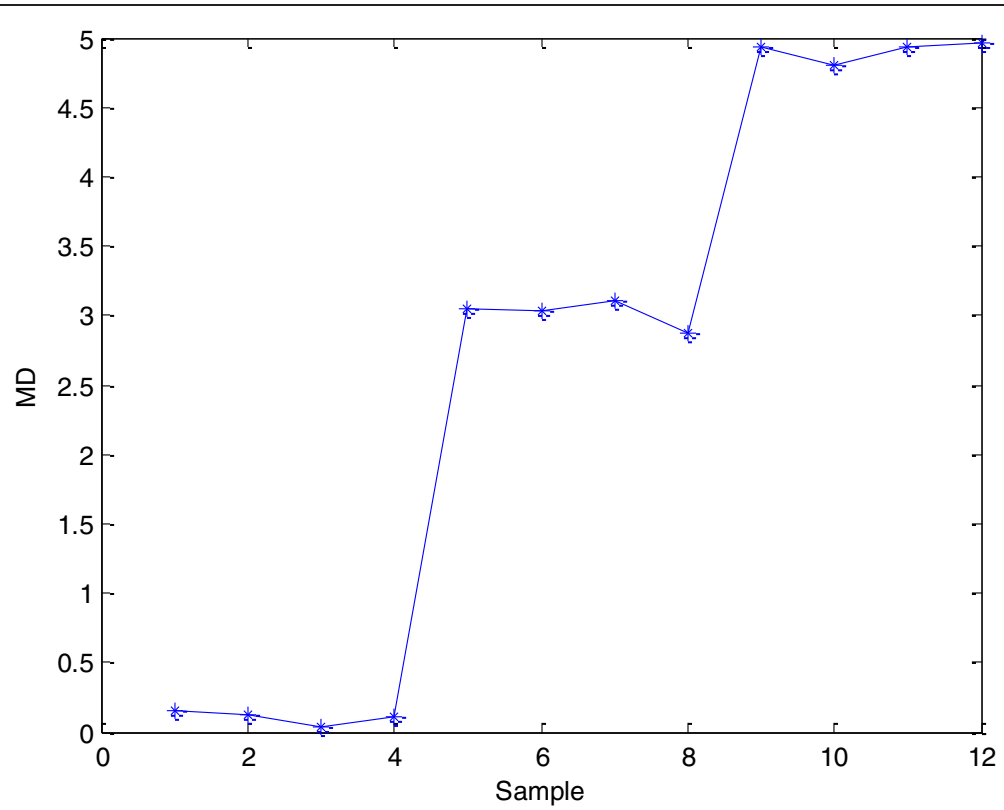

Figure $10 \mathrm{MD}$ result of testing set (hydraulic pump).

\subsubsection{Analysis of performance assessment results}

In this study, for the purpose of performance assessment, the normal samples numbered as Normal_1 to Normal_4 were used to construct and characterize the normal population noted as G_N. Through FDA, a space conversion method was achieved, by which, the different populations and the new measurement data can be projected from the original eight-dimensional space into a new two-dimensional space.
Then, the MDs between the normal population $G \_N$ and those samples included in the testing set, as shown in Table 2, were calculated. They were transformed into normalized CVs according to formula (13). The MD and CV curves are shown in Figures 10 and 11. Obviously, contrasting with the MDs of the normal testing samples, the MDs of the slipper loose and valve plate wear testing samples were quite large, because the normal testing samples were located nearby the normal population $G_{-} N$, while the

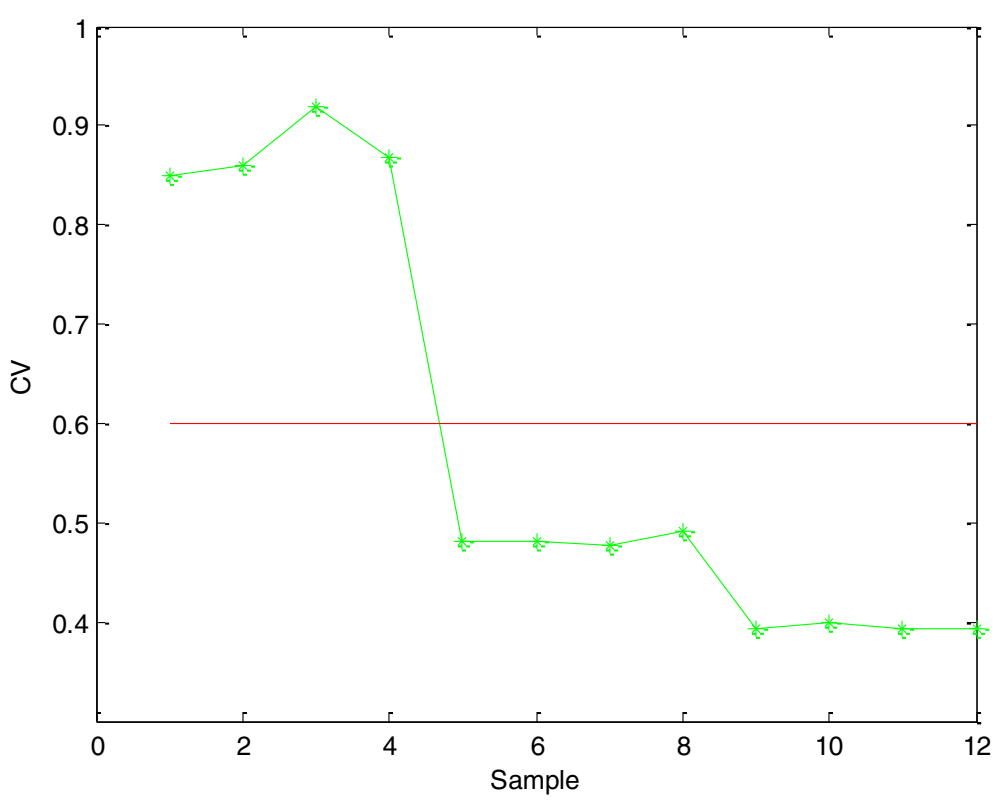

Figure $11 \mathrm{CV}$ result of testing set (hydraulic pump). 
Table 4 MDs and diagnosis results of samples in testing set (hydraulic pump)

\begin{tabular}{|c|c|c|c|c|c|c|c|c|c|c|c|c|}
\hline & N_1 & N_2 & N_3 & N_4 & F1_1 & F1_2 & F1_3 & F1_4 & F2_1 & F2_2 & F2_3 & F2_4 \\
\hline G_N & 0.1490 & 0.1288 & 0.0413 & 0.1135 & 3.0432 & 3.0394 & 3.1073 & 2.8714 & 4.9359 & 4.8058 & 4.9323 & 4.9614 \\
\hline G_F1 & 3.5555 & 3.5412 & 3.4477 & 3.5199 & 0.3838 & 0.3794 & 0.3107 & 0.5563 & 7.8717 & 7.7396 & 7.8727 & 7.9023 \\
\hline G_F2 & 4.7123 & 4.7364 & 4.8134 & 4.7444 & 7.4089 & 7.4290 & 7.5040 & 7.2451 & 0.0885 & 0.0495 & 0.0928 & 0.1209 \\
\hline Min & 0.1490 & 0.1288 & 0.0413 & 0.1135 & 0.3838 & 0.3794 & 0.3107 & 0.5563 & 0.0885 & 0.0495 & 0.0928 & 0.1209 \\
\hline Mode & G_N & G_N & G_N & G_N & G_F1 & G_F1 & G_F1 & G_F1 & G_F2 & G_F2 & G_F2 & G_F2 \\
\hline
\end{tabular}

fault testing samples were located far away, i.e., the slipper loose and valve plate wear samples were in an abnormal condition. Conversely, the CVs of the normal testing samples were relatively high; close to 0.9 . Whereas the CVs of the fault testing samples were all quite low; falling below the presupposed threshold of 0.6. Therefore, the CV index indicated that the normal testing samples were in a normal condition; while the slipper loose and valve plate wear testing samples were in a faulty condition. The analysis demonstrated that the performance assessment could be quantized and visualized by MD and CV, and coupled with a presupposed threshold where abnormal states can be detected. Therefore, this is a successful trial of the performance assessment and fault detection.

\subsubsection{Analysis of fault diagnosis results}

As previously mentioned in the performance assessment analysis, the samples in the testing set (numbered as F1_1 to F1_4 and F2_1 to F2_4) were detected as fault states. To identify which type of fault they belonged to, as a reference, the normal samples in the testing set (numbered as N_1 to N_4) were also considered. The MDs between those samples in the testing set and these three populations were calculated, as shown in Table 4 . In order to facilitate analysis, the normal population was noted as $G_{-} N$, the slipper loose and valve plate wear state populations were noted as G_F1 and G_F2, respectively. Through comparative analysis, the samples under normal conditions had the smallest MDs with the population $G_{-} N$, while the samples under slipper loose condition and valve plate wear condition had the smallest MDs with the population G_F1 and G_F2, respectively, as shown in the 'Min' row of Table 4. According to the discriminant rule in Section 2.5 , it can be determined that the samples N_1 to N_4 belonged to normal conditions, the samples F1_1 to F1_4 and F2_1 to F2_4 were under slipper loose state and valve plate wear state, respectively, as shown in the 'Mode' row. This accurate diagnosis result is further proof of the effectiveness of the proposed method in fault diagnosis.

\subsection{Performance assessment and fault diagnosis for ball bearing}

Bearings are critical components in rotating machines because their failure could lead to serious damage in machines. In recent years, bearing fault diagnosis has received increasing attention [34-36]. In this case, vibration signals are acquired from the ball bearing housing for performance assessment and fault diagnosis.

\subsubsection{Experimental setup and data acquisition}

In this case study, the test data were acquired from the Case Western Reserve University Bearing Data Center. As shown in Figure 12, the test-rig consists of a 2-horsepower motor (left), a torque transducer/encoder (center), a

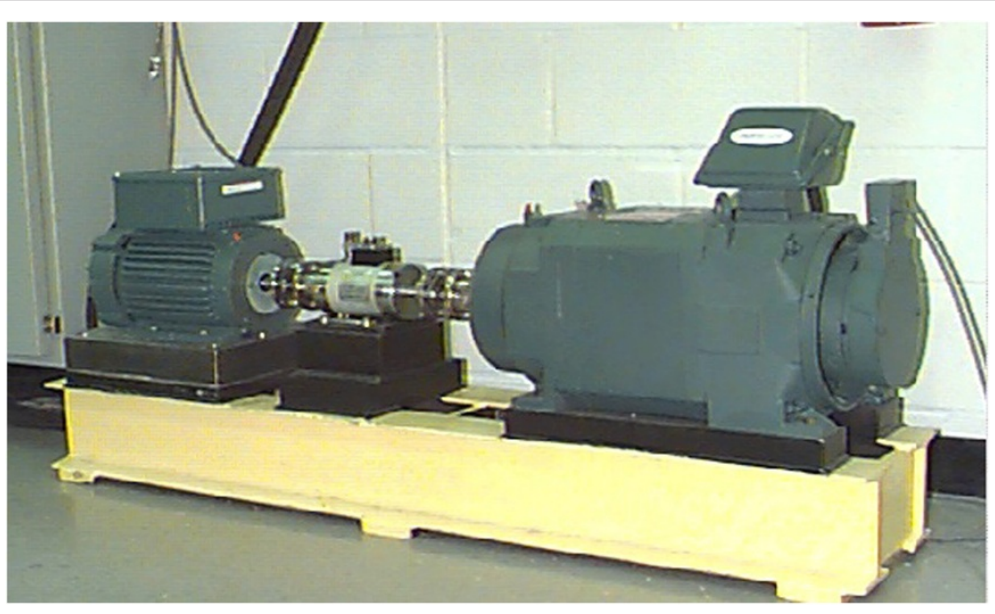

Figure 12 Test-rig of ball bearing. 
Table 5 Feature vector samples for learning (rolling bearing)

\begin{tabular}{lcccccccc}
\hline Number & $\mathbf{1}$ & $\mathbf{2}$ & $\mathbf{3}$ & $\mathbf{4}$ & $\mathbf{5}$ & $\mathbf{6}$ & $\mathbf{7}$ \\
\hline N_1 & 0.8111 & 0.5012 & 0.0524 & 0.2964 & 0.0002 & 0.0018 & 0.0053 & 0.0187 \\
\hline N_2 & 0.8259 & 0.4665 & 0.0544 & 0.3112 & 0.0002 & 0.0019 & 0.0054 & 0.02 \\
\hline N_3 & 0.7891 & 0.5274 & 0.0524 & 0.3097 & 0.0002 & 0.0018 & 0.0053 & 0.0204 \\
\hline N_4 & 0.8137 & 0.493 & 0.0553 & 0.3023 & 0.0002 & 0.0018 & 0.0056 & 0.0194 \\
\hline I_1 & 0.0795 & 0.2297 & 0.5852 & 0.1497 & 0.0014 & 0.0081 & 0.7446 & 0.1471 \\
\hline I_2 & 0.0823 & 0.2226 & 0.5831 & 0.1464 & 0.0015 & 0.0082 & 0.7467 & 0.157 \\
\hline I_3 & 0.081 & 0.2341 & 0.6031 & 0.1474 & 0.0014 & 0.0084 & 0.7306 & 0.1388 \\
\hline I_4 & 0.0723 & 0.2279 & 0.5922 & 0.1505 & 0.0014 & 0.0092 & 0.7412 & 0.1417 \\
\hline O_1 & 0.0069 & 0.0096 & 0.4907 & 0.0156 & 0.0082 & 0.0137 & 0.8681 & 0.0707 \\
\hline O_2 & 0.0065 & 0.0096 & 0.4945 & 0.0161 & 0.0081 & 0.0137 & 0.8655 & 0.0759 \\
\hline O_3 & 0.0069 & 0.0098 & 0.4831 & 0.0145 & 0.0076 & 0.0121 & 0.8725 & 0.0696 \\
\hline O_4 & 0.0065 & 0.01 & 0.5217 & 0.016 & 0.007 & 0.0124 & 0.8492 & 0.0777 \\
\hline B_1 & 0.045 & 0.0449 & 0.4876 & 0.0184 & 0.0005 & 0.0027 & 0.8703 & 0.0181 \\
\hline B_2 & 0.0443 & 0.0424 & 0.457 & 0.0189 & 0.0005 & 0.0025 & 0.887 & 0.0179 \\
\hline B_3 & 0.0426 & 0.0436 & 0.464 & 0.0187 & 0.0005 & 0.0025 & 0.8833 & 0.0186 \\
\hline B_4 & 0.0412 & 0.042 & 0.4584 & 0.0179 & 0.0004 & 0.0022 & 0.8865 & 0.0175 \\
\hline
\end{tabular}

dynamometer (right), and control electronics (data not shown). The test bearings support the motor shaft. Single point faults were introduced separately at the innerrace, outer-race, and rolling element (i.e., ball) of the test bearing using electro-discharge machining with fault diameters of $7 \mathrm{~mm}$. Faulted bearings were reinstalled into the test motor, and vibration data were recorded under different four states (normal, faults) using accelerometers, with motor loads of 2 horsepower, motor speed of $1750 \mathrm{rpm}$, and a sampling frequency of $12,000 \mathrm{~Hz}$.

\subsubsection{Feature extraction by WPA}

Through implementation of FFT on the acquired vibration data, it was found that the characteristic frequencies under normal, inner-race fault, outer-race fault, and ball fault states are distributed separately in different frequency bands, and that there is little overlap between any two adjacent frequencies in the spectrum. Therefore, three-layer wavelet packet decomposition can also be applied to the vibration data, and thus, eight-dimensional feature vectors can be acquired by calculating and normalizing the energy of each band.

Table 6 Feature vector samples for testing (rolling bearing)

\begin{tabular}{|c|c|c|c|c|c|c|c|c|}
\hline Number & 1 & 2 & 3 & 4 & 5 & 6 & 7 & 8 \\
\hline N_T1 & 0.804 & 0.5112 & 0.0511 & 0.2987 & 0.0002 & 0.0016 & 0.0051 & 0.0192 \\
\hline N_T2 & 0.8119 & 0.5007 & 0.0528 & 0.2948 & 0.0002 & 0.0018 & 0.0053 & 0.019 \\
\hline N_T3 & 0.8208 & 0.4824 & 0.0538 & 0.3006 & 0.0002 & 0.0017 & 0.0055 & 0.0194 \\
\hline N_T4 & 0.7854 & 0.5344 & 0.0532 & 0.307 & 0.0002 & 0.0018 & 0.0054 & 0.0198 \\
\hline I_T1 & 0.0734 & 0.2138 & 0.58 & 0.1423 & 0.0015 & 0.008 & 0.7556 & 0.1461 \\
\hline I_T2 & 0.087 & 0.2276 & 0.5929 & 0.15 & 0.0014 & 0.0089 & 0.7394 & 0.1403 \\
\hline I_T3 & 0.0769 & 0.2242 & 0.6017 & 0.1463 & 0.0016 & 0.0084 & 0.7343 & 0.1454 \\
\hline I_T4 & 0.071 & 0.2271 & 0.5888 & 0.1535 & 0.0014 & 0.0082 & 0.7421 & 0.1498 \\
\hline O_T1 & 0.0066 & 0.0091 & 0.4939 & 0.0159 & 0.0073 & 0.0132 & 0.8661 & 0.0736 \\
\hline O_T2 & 0.0073 & 0.0098 & 0.4945 & 0.0166 & 0.0075 & 0.0128 & 0.8655 & 0.0759 \\
\hline O_T3 & 0.0068 & 0.0108 & 0.5057 & 0.0159 & 0.0073 & 0.0137 & 0.8594 & 0.0706 \\
\hline O_T4 & 0.0069 & 0.0098 & 0.4976 & 0.0154 & 0.0077 & 0.0133 & 0.8642 & 0.0705 \\
\hline B_T1 & 0.0438 & 0.0431 & 0.483 & 0.0187 & 0.0005 & 0.0024 & 0.8731 & 0.0188 \\
\hline B_T2 & 0.0438 & 0.0439 & 0.4753 & 0.018 & 0.0006 & 0.0026 & 0.8773 & 0.018 \\
\hline B_T3 & 0.046 & 0.0411 & 0.471 & 0.0197 & 0.0005 & 0.0023 & 0.8796 & 0.0176 \\
\hline B_T4 & 0.043 & 0.0436 & 0.4627 & 0.0187 & 0.0005 & 0.0023 & 0.884 & 0.0174 \\
\hline
\end{tabular}




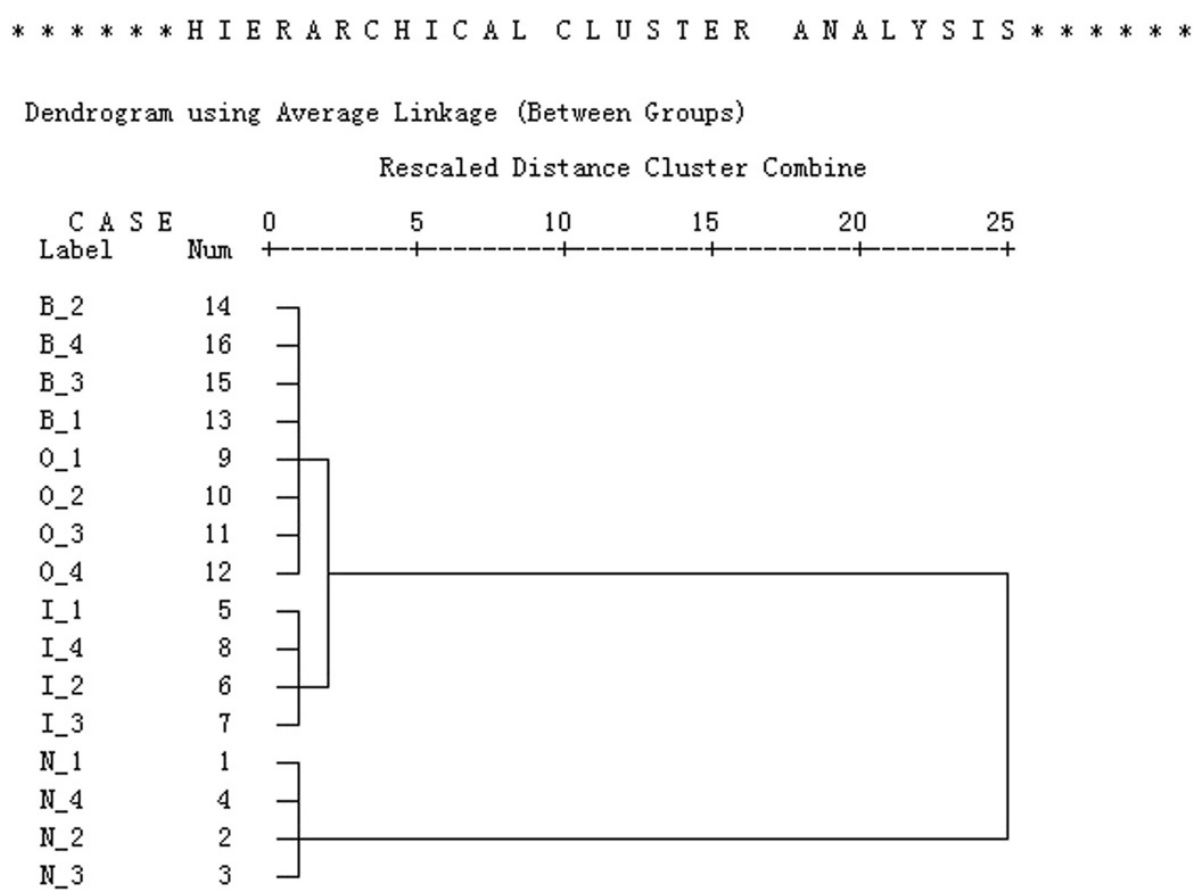

Figure $13 \mathrm{~A}$ tree dendrogram of HCA (rolling bearing).

For those four states, eight feature vector samples were acquired. The first four samples of each state were used as the FDA learning set (for clearer identification they were numbered as N_1 to N_4, I_1 to I_4, O_1 to O_4, and B_1 to $\mathrm{B} \_4$, corresponding to the normal state, inner-race fault state, outer-race fault state, and ball fault state, respectively), while the others were used as the testing set (they were also numbered in the same manner as $\mathrm{N}_{-} \mathrm{T} 1$ to N_T4, I_T1 to I_T4, O_T1 to O_T4, and B_T1 to B_T4, respectively).

The feature vector samples for learning and testing are shown in Tables 5 and 6.

\subsubsection{Analysis of states separability}

The HCA was applied to the learning set, as shown in Figure 13, and the whole process of clustering was displayed in a tree dendrogram. First, each sample was taken as a class. After the first clustering, according to the distance between classes, samples B_1 to B_4 and samples O_1 to O_4 were merged into a cluster, samples I_1 to I_4 were merged into another cluster, and samples $\mathrm{N} \_1$ to N_4 were merged into the third cluster. As shown in Table 7, the cluster membership was that all the normal samples gathered in cluster 1 , all the inner-race fault samples gathered in cluster 2 , all the outer-race fault samples gathered in cluster 3, and all the ball fault samples gathered in cluster 4. The outputs of SPSS Statistics, the tree dendrogram, and the cluster membership table successfully demonstrated the separability of feature vector samples acquired during those four conditions.

\subsubsection{Analysis of performance assessment results}

In this study, the normal samples numbered $N_{-} 1$ to $N_{-} 4$ were used to construct and characterize the normal population noted as G_N. Through FDA, the original

Table 7 Clustering memberships of learning set (rolling bearing)

\begin{tabular}{|c|c|}
\hline Case & Cluster index \\
\hline $1: N_{-} 1$ & 1 \\
\hline $2: N \_2$ & 1 \\
\hline $3: N_{-} 3$ & 1 \\
\hline $4: N \_4$ & 1 \\
\hline 5:I_1 & 2 \\
\hline $6: 1 \_2$ & 2 \\
\hline $7: 1 \_3$ & 2 \\
\hline $8: 1 \_4$ & 2 \\
\hline 9:O_1 & 3 \\
\hline 10:0_2 & 3 \\
\hline $11: 0 \_3$ & 3 \\
\hline 12:O_4 & 3 \\
\hline 13:B_1 & 4 \\
\hline 14:B_2 & 4 \\
\hline 15:B_3 & 4 \\
\hline 16:B_4 & 4 \\
\hline
\end{tabular}




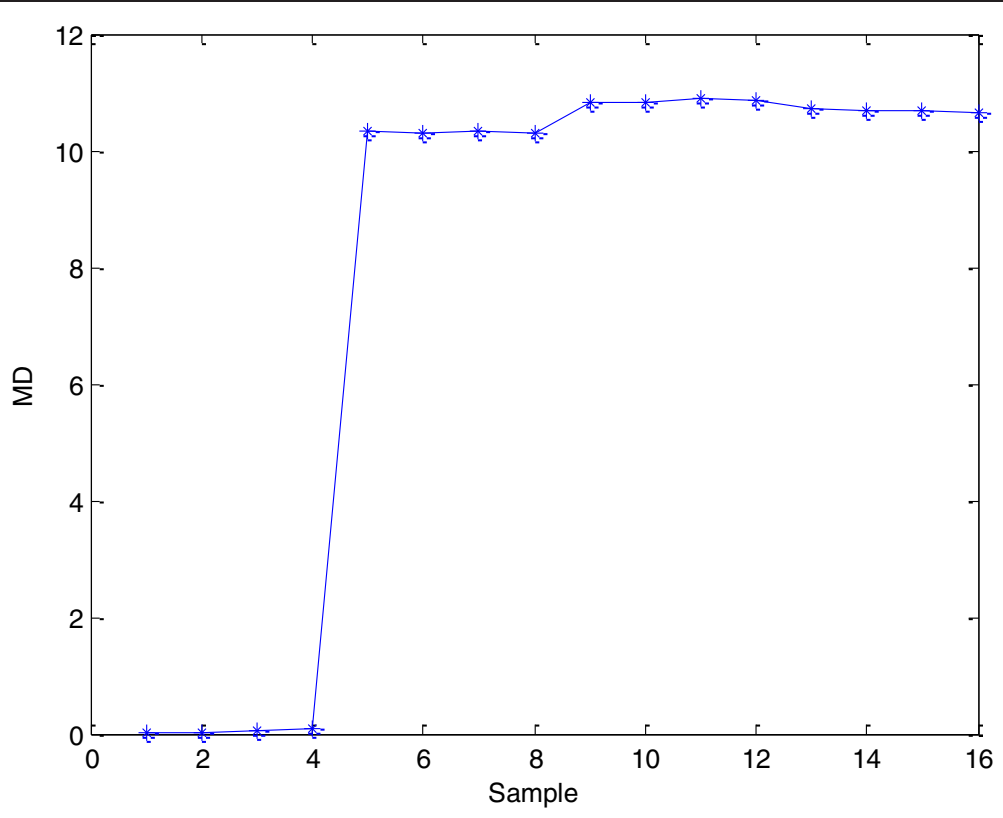

Figure $14 \mathrm{MD}$ result of testing set (rolling bearing).

eight-dimensional space was projected into a new threedimensional space.

The MDs between the normal population $G_{-} N$ and those samples included in the testing set, as shown in Table 6, were calculated and transformed into normalized CVs according to formula (13). The MD and CV curves are shown in Figures 14 and 15. Apparently, the MDs of the normal testing samples were quite low, while those of the fault testing samples were quite large. Because the normal testing samples were located nearby the normal population $G \_N$, while the inner-race fault, outer-race fault, and ball fault testing samples were located far away. Conversely, the CVs of the normal samples were relatively large, whereas the CVs of all fault testing samples were quite low, falling below the presupposed threshold of 0.6 . Thus, it indicated that the inner-race fault, outer-race fault, and ball fault samples were in a faulty condition. Therefore, this result demonstrated that the performance assessment of ball bearing can be quantized and visualized by $\mathrm{MD}$ and $\mathrm{CV}$.

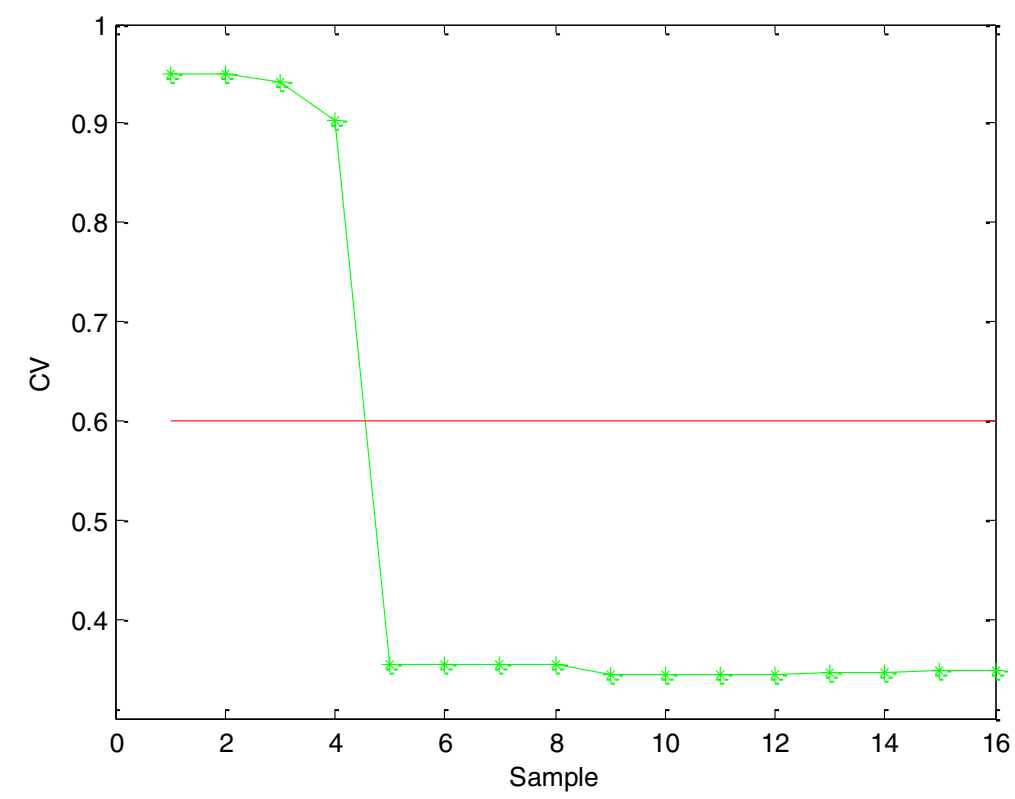

Figure $15 \mathrm{CV}$ result of testing set (rolling bearing). 
Table 8 MDs and diagnosis results of samples in testing set (rolling bearing)

\begin{tabular}{|c|c|c|c|c|c|c|c|c|}
\hline & N_T1 & N_T2 & N_T3 & N_T4 & I_T1 & I_T2 & I_T3 & I_T4 \\
\hline G_N & 0.0245 & 0.0249 & 0.0352 & 0.1014 & 10.3384 & 10.3041 & 10.3426 & 10.2988 \\
\hline G_I & 10.3123 & 10.3094 & 10.3010 & 10.3022 & 0.1079 & 0.0547 & 0.0639 & 0.0609 \\
\hline G_O & 10.8671 & 10.8645 & 10.8462 & 10.8709 & 1.8074 & 1.9666 & 1.8590 & 1.8561 \\
\hline G_B & 10.6774 & 10.6747 & 10.6590 & 10.6777 & 1.2993 & 1.4575 & 1.3519 & 1.3486 \\
\hline Min & 0.0245 & 0.0249 & 0.0352 & 0.1014 & 0.1079 & 0.0547 & 0.0639 & 0.0609 \\
\hline \multirow[t]{2}{*}{ Mode } & G_N & G_N & G_N & G_N & G_I & G_I & G_I & G_I \\
\hline & O_T1 & O_T2 & O_T3 & O_T4 & B_T1 & B_T2 & B_T3 & B_T4 \\
\hline G_N & 10.8419 & 10.8391 & 10.8812 & 10.8567 & 10.7244 & 10.6955 & 10.6792 & 10.6498 \\
\hline G_l & 1.9145 & 1.9065 & 1.9045 & 1.9098 & 1.4065 & 1.3990 & 1.4010 & 1.4045 \\
\hline G_O & 0.0122 & 0.0141 & 0.0358 & 0.0078 & 0.5250 & 0.5308 & 0.5288 & 0.5272 \\
\hline G_B & 0.5243 & 0.5168 & 0.5168 & 0.5198 & 0.0642 & 0.0362 & 0.0181 & 0.0150 \\
\hline Min & 0.0122 & 0.0141 & 0.0358 & 0.0078 & 0.0642 & 0.0362 & 0.0181 & 0.0150 \\
\hline Mode & G_O & G_O & G_O & G_o & G_B & G_B & G_B & G_B \\
\hline
\end{tabular}

\subsubsection{Analysis of fault diagnosis results}

In the performance assessment analysis, the CVs of the samples in the testing set (numbered as I_T1 to I_T4, O_T1 to O_T4, and B_T1 to B_T4) were quite low, so they were in fault states. To identify which type of fault they belonged to, as a reference, the normal samples in the testing set (numbered as N_T1 to N_T4) were also considered, the MDs between the samples in the testing set and those four populations were calculated, as shown in Table 8. In order to facilitate analysis, the normal population was noted as $G_{-} N$, the inner-race-fault, outer-race-fault, and ball fault populations were noted as G_I, G_O, and G_B, respectively. Through comparative analysis, the samples under normal conditions had the smallest MDs with the population $G_{-} N$, while the samples under inner-race-fault, outer-race-fault, and ball fault conditions had the smallest MDs with the population G_I, G_O, and G_B, respectively, as shown in the 'Min' row of Table 8. Therefore, it can be determined that the samples $\mathrm{N}_{-} \mathrm{T} 1$ to $\mathrm{N}_{-} \mathrm{T} 4$ belonged to normal condition, the samples I_T1 to I_T4, O_T1 to O_T4, and $\mathrm{B} \_\mathrm{T} 1$ to $\mathrm{B} \_\mathrm{T} 4$ were under inner-race fault condition, outer-race fault condition, and ball fault condition, respectively, as shown in the 'Mode' row. This accurate diagnosis result is further proof of the effectiveness of the proposed method in fault diagnosis.

\section{Conclusion}

In this article, addressing the challenging issues on performance assessment, fault detection, and fault diagnosis, a novel method based on FDA and MD is introduced, and an integrated framework for performance assessment, fault detection, and fault diagnosis is built.

In this method, FDA is applied as an optimal linear dimensionality reduction technique, in terms of maximizing the separation between different populations. In the new low-dimensional space, MD, which can be transformed into normalized $\mathrm{CV}$, is calculated for performance assessment, and abnormal states can be detected with the presupposed threshold. Furthermore, once various fault data are available, the unknown fault mode can be identified accurately by comparing the MDs between the new data and each normal/fault population.

However, how to transform MD into $\mathrm{CV}$ for performance assessment and to determine an adaptive threshold for fault detection is still a challenge for future work. In the future, we are going to acquire sequential online degradation measurements for real-time performance degradation assessment and detection, and enlarge the number of fault and learning samples for more accurate fault diagnosis. Moreover, we plan to apply this approach to different components, such as gearboxes, shafts, etc., to further verify the effectiveness and evaluate the possibility of generalizing the proposed approach.

\section{Competing interests}

The authors declare that they have no competing interests.

\section{Authors' contributions}

$X C T$ carried out the performance assessment studies, and drafted the manuscript; CL (Corresponding author) carried out the diagnosis studies, and participated in the algorithm design and manuscript revision; $C L$ and ZLW carried out the preparation of experimental data, and participated in the algorithm design. All authors read and approved the final manuscript.

\section{Acknowledgements}

This research was supported by the National Natural Science Foundation of China (Grant nos.61074083, 50705005, and 51105019) as well as the Technology Foundation Program of National Defense (Grant no.

Z132010B004). The authors are very grateful for the valuable suggestions from the reviewers and editor.

Received: 7 May 2012 Accepted: 2 December 2012

Published: 10 January 2013 


\section{References}

1. J Lee, J Ni, D Djurdjanovic, H Qiu, HT Liao, Intelligent prognostics tools and e-maintenance. Comput. Ind. 57, 476-489 (2006). doi:10.1016/j. compind.2006.02.014

2. AKS Jardine, D Lin, D Banjevic, A review on machinery diagnostics and prognostics implementing condition-based maintenance. Mech. Syst. Signal Process. 20, 1483-1510 (2006). doi:10.1016/j.ymssp. 2005.09.012

3. A Heng, S Zhang, ACC Tan, J Mathew, Rotating machinery prognostics: state of the art, challenges and opportunities. Mech. Syst. Signal Process. 23, 724-739 (2009). doi:10.1016/j.ymssp. 2008.06.009

4. HP Bloch, FK Geitner, Practical Machinery Management for Process Plants. Machinery Failure Analysis and Trouble Shooting, vol. 2, 3rd edn. (Gulf Professional Publishing, Houston, 1997)

5. WB Wang, Modelling the probability assessment of system state using available condition information. IMA J. Manage. Math. 17(3), 225-234 (2006). doi:10.1093/imaman/dpi035

6. WB Wang, A two-stage prognosis model in condition based maintenance. Eur. J. Oper. Res. 182, 1177-1187 (2007). doi:10.1016/j.ejor.2006.08.047

7. LL Ma, Z Zhang, JZ Wang, Combination method of support vector machine and fisher discriminant analysis for chemical process fault diagnosis, in Paper presented at the 29th Chinese Control Conference (Beijing, 2010), pp. 4000-4003

8. LH Chiang, ME Kotanchek, AK Kordon, Fault diagnosis based on Fisher discriminant analysis and support vector machines. Comput. Chem. Eng. 28, 1389-1401 (2004). doi:10.1016/j.compchemeng.2003.10.002

9. QP He, SJ Qin, J Wang, A new fault diagnosis method using fault directions in Fisher discriminant analysis. AICHE J. 51(2), 555-571 (2005). doi:10.1002/aic.10325

10. XB He, W Wang, YP Yang, YH Yang, Variable-weighted Fisher discriminant analysis for process fault diagnosis. J. Process. Control. 19, 923-931 (2009). doi:10.1016/j.jprocont.2008.12.001

11. MJ Fuente, D Garcia-Alvarez, GI Sainz-Palmero, Fault detection and identification method based on multivariate statistical techniques, in Paper presented at the Proceedings of Emerging Technologies \& Factory Automation (Mallorca, 2009), pp. 1-6

12. LX Liao, J Lee, Design of a reconfigurable prognostics platform for machine tools. Expert Syst. Appl. 37, 240-252 (2010). doi:10.1016/j.eswa.2009.05.004

13. I Gurrutxaga, I Albisua, O Arbelaitz, JI Martin, J Muguerza, JM Perez, I Perona, An efficient method to find the best partition in hierarchical clustering based on a new cluster validity index. Pattern Recognit 43, 3364-3373 (2010). doi:10.1016/j.patcog.2010.04.021

14. J Goldberger, T Tassa, A hierarchical clustering algorithm based on the Hungarian method. Pattern Recognit. Lett. 29(11), 1632-1638 (2008) doi:10.1016/j.patrec.2008.04.003

15. M Safayani, MTM Shalman, Matrix-variate probabilistic model for canonical correlation analysis. EURASIP J. Adv. Signal Process. 7, (2011). Article ID 748430

16. A Eftekhari, HA Moghaddam, M Forouzanfar, J Alirezaie, Incremental local linear fuzzy classifier in fisher space. EURASIP J. Adv. Signal Process., (2009). Article ID 360834

17. LX Liao, An Adaptive Modeling for Robust Prognostics on a Reconfigurable Platform. PhD, University of Cincinnati, Engineering: Industrial Engineering (University of Cincinnati, Cincinnati, 2010)

18. XZ Zhao, BY Ye, Convolution wavelet packet transform and its applications to signal processing. Dig. Signal Process. 20, 1352-1364 (2010). doi:10.1016/j. dsp. 2010.01.007

19. JD Wu, CH Liu, An expert system for fault diagnosis in internal combustion engines using wavelet packet transform and neural network. Expert Syst. Appl. 36, 4278-4286 (2009). doi:10.1016/j.eswa.2008.03.008

20. YN Pan, J Chen, XL Li, Bearing performance degradation assessment based on lifting wavelet packet decomposition and fuzzy c-means. Mech. Syst. Signal Process. 24, 559-566 (2010). doi:10.1016/j.ymssp. 2009.07.012

21. B Kotnik, Z Kacic, A comprehensive noise robust speech parameterization algorithm using wavelet packet decomposition-based denoising and speech feature representation techniques. EURASIP J. Adv. Signal Process., (2007). Article ID 64102

22. G Forestier, C Wemmert, P Gancarski, Multisource images analysis using collaborative clustering. EURASIP J. Adv. Signal Process., (2008). Art ID 374095

23. ZS Wang, A Maier, NK Logothetis, HL Liang, Single-trial classification of bistable perception by integrating empirical mode decomposition, clustering, and support vector machine. EURASIP J. Adv. Signal Process., (2008). Article ID 592742

24. I Kojadinovic, Hierarchical clustering of continuous variables based on the empirical copula process and permutation linkages. Comput. Stat. Data Anal. 54, 90-108 (2010). doi:10.1016/j.csda.2009.07.014

25. JAS Almeida, LMS Barbosa, AACC Pais, SJ Formosinho, Improving hierarchical cluster analysis: a new method with outlier detection and automatic clustering. Chemometrics Int. Lab. Syst. 87, 208-217 (2007). doi:10.1016/j.chemolab.2007.01.005

26. ML Song, YQ Song, HY Yu, ZY Wang, Calculation of China's environmenta efficiency and relevant hierarchical cluster analysis from the perspective of regional differences. Math. Comput. Model., (2012). doi:10.1016/j.mcm. 2012.04.003

27. C Bouveyron, C Brunet, Probabilistic Fisher discriminant analysis: a robust and flexible alternative to Fisher discriminant analysis. Neurocomputing 90, 12-22 (2012). doi:10.1016/j.neucom.2011.11.027

28. R Khemchandani, Jayadeva, S Chandra, Learning the optimal kernel for Fisher discriminant analysis via second order cone programming. Eur. J. Oper. Res. 203, 692-697 (2010). doi:10.1016/j.ejor.2009.09.020

29. H Shin, An extension of Fisher's discriminant analysis for stochastic processes. J. Multivariate Anal. 99, 1191-1216 (2008). doi:10.1016/j. jmva.2007.08.001

30. CR Rao, Linear Statistical Inference and Its Application, 2nd edn. (Wiley, New York, 1973)

31. A Heng, A Tan, J Mathew, BS Yang, Machine prognosis with full utilization of truncated lifetime data, in Proceedings of the Second World Congress on Engineering Asset Management (, Harrogate, 2007), pp. 775-784

32. G Niu, S Singh, SW Holland, M Pecht, Health monitoring of electronic products based on Mahalanobis distance and Weibull decision metrics. Microelectron. Reliab. 51, 279-284 (2011). doi:10.1016/j.microrel.2010.09.009

33. JP Wang, HT Hu, Vibration-based fault diagnosis of pump using fuzzy technique. Measurement 39, 176-185 (2006). doi:10.1016/j. measurement.2005.07.015

34. S Prabhakar, AR Mohanty, AS Sekhar, Application of discrete wavelet transform for detection of ball bearing race faults. Tribol. Int. 35(12), 793-800 (2002)

35. KF Al-Raheem, A Roy, KP Ramachandran, DK Harrison, S Grainger, Rolling element bearing fault diagnosis using Laplace-Wavelet envelope power spectrum. EURASIP J. Adv. Signal Process., (2007). Article ID 073629

36. A Ibrahim, F Bonnardot, M El Badaoui, F Guillet, Detection of bearing damage using stator current, and voltage to cancel electrical noise. EURASIP J. Adv. Signal Process., (2011). Article ID 235236

doi:10.1186/1687-6180-2013-5

Cite this article as: Tao et al:: An approach to performance assessment and fault diagnosis for rotating machinery equipment. EURASIP Journal on Advances in Signal Processing 2013 2013:5.

\section{Submit your manuscript to a SpringerOpen ${ }^{\odot}$ journal and benefit from:}

- Convenient online submission

Rigorous peer review

- Immediate publication on acceptance

- Open access: articles freely available online

- High visibility within the field

- Retaining the copyright to your article

Submit your next manuscript at $>$ springeropen.com 